\title{
Chondroprotective Effects of Hyaluronic Acid-Chitosan Nanoparticles Containing Plasmid DNA Encoding Cytokine Response Modifier A in a Rat Knee Osteoarthritis Model
}

\author{
Pang-hu Zhou ${ }^{a}$ Bo Qiu ${ }^{a}$ Rong-hui Deng ${ }^{a}$ Hua-jie Lia Xiong-feng Xu ${ }^{a}$ \\ Xi-fu Shang ${ }^{\text {b }}$ \\ aDepartment of Orthopedics, Renmin Hospital of Wuhan University, Wuhan, ${ }^{\mathrm{b}}$ Department of \\ Orthopedics, Anhui Provincial Hospital, Hefei, China
}

\section{Key Words}

Cytokine response modifier A $\cdot$ Hyaluronic acid $\cdot$ Chitosan $•$ Nanoparticles $•$ Gene delivery • Osteoarthritis

\begin{abstract}
Background/Aims: Interleukin (IL)-1 $\beta$ plays an essential role in the pathophysiology of osteoarthritis (OA). Cytokine response modifier $\mathrm{A}(\mathrm{CrmA})$ can prevent the generation of active IL-1 $\beta$. This study aimed to explore the chondroprotective effects of hyaluronic acid-chitosan nanoparticles containing plasmid DNA encoding $\mathrm{CrmA}(\mathrm{HA} / \mathrm{CS}-\mathrm{CrmA})$ in a rat OA model. Methods: HA/CS-CrmA nanoparticles were synthesized through the complex coacervation of cationic polymers. The characteristics, toxicity, and transfection of the nanoparticles were investigated. Furthermore, the potential effects of HA/CS-CrmA nanoparticles were evaluated via a rat anterior cruciate ligament transection (ACLT) model of OA. Cartilage damage and synovial inflammation were assessed by safranin O/fast green and hematoxylin and eosin staining. Type II collagen in cartilage was measured by immunohistochemistry, and the expression levels of IL-1 $\beta$, matrix metalloproteinase (MMP)-3, and MMP-13 in synovial tissue were detected by western blot. Results: The HA/CS-CrmA nanoparticles, which effectively entrapped plasmid DNA, showed an adequate size (100-300 nm) and a regular spherical shape. The nanoparticles safely transfected synoviocytes and released plasmid DNA in a sustained manner over 3 weeks. Additionally, HA/CS-CrmA nanoparticles significantly inhibited cartilage damage, synovial inflammation, and the loss of type II collagen induced by ACLT. The expression levels of IL-1 $\beta$, MMP-3, and MMP-13 in synovial tissue were dramatically down-regulated by HA/CS-CrmA nanoparticles. Conclusions: These results suggested that HA/CS-CrmA nanoparticles could attenuate cartilage destruction and protect against early OA by inhibiting synovial inflammation via inhibition of IL-1 $\beta$ generation.

P.-h. Zhou and B. Qiu contributed equally to this work. 


\section{Introduction}

Osteoarthritis $(\mathrm{OA})$, the most prevalent disease of articular joints, is a leading cause of chronic pain and disability worldwide in elderly population [1]. OA is a complex pathophysiological process in which inflammation plays an important role [2,3]. Specifically, synovial inflammation contributes to the pathogenesis and progression of OA at an early stage of the disease [4]. Inflamed synoviocytes secrete pro-inflammatory and catabolic factors, such as interleukin (IL)-1 $\beta$, IL-6, tumor necrosis factor $\alpha$, and matrix metalloproteinases (MMPs), which promote cartilage inflammation, apoptosis and degradation [2, 5, 6]. Among those mediators, IL- $1 \beta$ is considered a key target because it induces the production of MMPs and the degradation of type II collagen, and is involved in the transmission of pain [7-10]. Therefore, it has been proposed that inhibition of the IL-1 $\beta$ pathway in the synovium could prevent the degradation of joint cartilage during the pathogenesis of OA.

Cytokine response modifier A (CrmA), a cowpox virus-encoded serpin-like protease inhibitor, can tightly bind to IL-1 $\beta$ converting enzyme (caspase-1) and consequently inhibit the generation of IL-1 $\beta[11,12]$. Thus, CrmA might exert potential therapeutic effects on OA. Our previous study revealed that the controlled release of CrmA from chitosan (CS) microspheres could suppress chondrocyte inflammation and apoptosis induced by IL-1 $\beta$ in vitro [13]. However, the effects of CrmA on OA-related synovitis and cartilage damage in vivo remain unknown. Meanwhile, the relatively rapid clearance and short biological half-life of the protein limit the application of CrmA-loaded CS microspheres. Thus, a new strategy should be developed to prolong the effectiveness of CrmA.

Gene therapy, which targets specific pathological mechanisms of OA and continuously produces targeted therapeutic proteins, is a promising treatment for OA [14]. A key issue of gene therapy is the selection of an effective and appropriate gene delivery vector. Compared with most commonly used viral vectors, non-viral gene delivery systems are considered safer alternatives because they are more stable in storage and can be administered repeatedly with less host immune response [15]. CS, a natural polysaccharide with a structure similar to glycosaminoglycans (GAGs), has emerged as a widely used non-viral gene delivery vector due to its non-toxicity, good biocompatibility, biodegradability, and high stability $[16,17]$. Hyaluronic acid (HA) is another biocompatible anionic biopolymer used in a wide array of clinical applications $[18,19]$. As a natural GAG of the synovial fluid and extracellular matrix of articular cartilage, HA contributes to the elasticity and viscosity of synovial fluid and plays an important role in cell adhesion, morphogenesis, and the regulation of inflammation [18]. Moreover, HA can bind cluster determinant 44 (CD44) [20], which is highly expressed in chondrocytes and synoviocytes in OA [21-23]. A previous study demonstrated that HACS nanoparticles could be effective non-viral vectors suitable for the delivery of genes to chondrocytes [24].

Accordingly, we designed a non-viral vector composed of HA-CS to deliver the target CrmA gene and evaluated the characteristics, toxicity, and transfection of the synthesized nanoparticles in vitro. We subsequently assessed the therapeutic effects in a rat OA model constructed by anterior cruciate ligament transection (ACLT) in vivo.

\section{Materials and Methods}

Ethics Statement

All procedures involving animals in the present study conformed to the Guideline for the Care and Use of Laboratory Animals published by the US National Institutes of Health and were approved by Institutional Animal Care and Use Committee of Wuhan University (Approval number: 2016-0138).

Fabrication of Nanoparticles Loaded with pDNA encoding CrmA

Plasmid DNA containing the CrmA sequence (pDNA-CrmA) was synthesized by BioVector, Inc. (Beijing, China). The pDNA-CrmA was propagated in Escherichia coli cells prior to isolation and purification. The 
absorption ratio was measured at $\lambda=260 \mathrm{~nm}$ and $280 \mathrm{~nm}$ to evaluate the concentration and purity of the plasmid. The same plasmid vector without the CrmA sequence was used an empty plasmid control.

HA/CS nanoparticles loaded with pDNA-CrmA (HA/CS-CrmA) were prepared according to the method described previously [25, 26]. Briefly, 2 g CS (molecular weight: $150 \mathrm{kDa}$, deacetylation: 98\%, Sigma-Aldrich, St. Louis, USA) was dissolved in $100 \mathrm{~mL}$ of acetic acid ( $\mathrm{pH} 5.5$ ) with vigorous stirring at $-20^{\circ} \mathrm{C}$ for $3 \mathrm{~h}$; the $1 \%$ HA (molecular weight: 500-730 kDa, Sigma-Aldrich) solution was acquired using the same method. The HA and CS solutions at a volume ratio of 1:2 were mixed under magnetic stirring. The required volume of pDNACrmA was gently pipetted into the HA/CS solution with a theoretical loading of $10 \%(\mathrm{w} / \mathrm{w})$. The resulting mixture was rapidly vortexed for 3-5 s and left for $1 \mathrm{~h}$ at room temperature to allow the complexes to form completely. Nanoparticle samples were centrifuged at $10,000 \mathrm{rpm}$ for $30 \mathrm{~min}$ at $4{ }^{\circ} \mathrm{C}$, and maintained for 24 $\mathrm{h}$ at $-80{ }^{\circ} \mathrm{C}$, followed by vacuum lyophilization. Lyophilized nanoparticle samples were stored at $4{ }^{\circ} \mathrm{C}$ until the experiment. The HA/CS nanoparticles without pDNA-CrmA were used as an empty nanoparticle control.

\section{Nanoparticle Characterization}

Morphology of the Nanoparticles. The morphology of the HA/CS-CrmA nanoparticles was measured by scanning electron microscope (SEM; JSM-6330; JEOL, Tokyo, Japan). The HA/CS-CrmA nanoparticle solution was dropped on a clean glass slide and dried at $37^{\circ} \mathrm{C}$ overnight. The slide was then coated with gold and observed by SEM.

Gel-Retarding Analysis. The degree of protection afforded by HA/CS on pDNA-CrmA was evaluated by agarose gel electrophoresis assays. The HA/CS-CrmA and naked pDNA-CrmA samples were loaded onto a $1 \%$ agarose gel containing ethidium bromide in Tris-borate EDTA buffer (pH 8.0) and run on the gel at 120 $\mathrm{V}$ for $30 \mathrm{~min}$. HA/CS-CrmA and naked pDNA-CrmA solutions both containing $1 \mu \mathrm{g}$ pDNA were added to $5 \mathrm{U}$ DNase I (Sigma-Aldrich) and maintained in a water bath at $37^{\circ} \mathrm{C}$ for $1 \mathrm{~h}$. Meanwhile, chitosanase $(0.7 \mathrm{U} / \mathrm{mg}$ of nanoparticles, Sigma-Aldrich) were added to another HA/CS-CrmA solution and kept in a water bath at $37^{\circ} \mathrm{C}$ for $4 \mathrm{~h}$. Then, $1 \%$ agarose gel electrophoresis was repeated, as described above.

In vitro $p D N A$ Release Study. The quantity of pDNA-CrmA release from the HA/CS-CrmA nanoparticles was measured by dissolving HA/CS-CrmA nanoparticles in phosphate-buffered saline (pH 7.4) at $37{ }^{\circ} \mathrm{C}$ in a shaker bath at $135 \mathrm{rpm}$. At appropriate time intervals $(1,4,7,10,13,16,19$, and 22 days), samples were centrifuged to collect the supernatants for analysis. The amount of pDNA-CrmA released into the supernatant was determined by spectrophotometer (DU640; Beckman, Fullerton, CA, USA) at $260 \mathrm{~nm}$. The supernatant of HA/CS nanoparticles without pDNA-CrmA was used as control to normalize the absorption at $260 \mathrm{~nm}$.

\section{Cell Studies}

Isolation and Culture of Synoviocytes. Synovial tissues were obtained under sterile conditions from the knee joints of 6-8 weeks old Sprague-Dawley rats (Experimental and Animal Center of Wuhan University, Wuhan, China). Before isolating the synovial tissues, the rats were euthanized with an overdose of intraperitoneal injection of sodium pentobarbital (Sigma-Aldrich) [27]. Synovial tissues were cut into small pieces $\left(<1 \mathrm{~m}^{3}\right.$ ) and digested with $0.2 \%$ collagenase I (Gibco, Carlsbad, CA, USA) for $3 \mathrm{~h}$. After washing twice with DMEM, the isolated synoviocytes were cultured in monolayers in culture medium composed of DMEM/ F12 (Gibco) supplemented with $10 \%$ fetal bovine serum and $1 \%$ antibiotics at $37{ }^{\circ} \mathrm{C}$ with $5 \% \mathrm{CO}_{2}$.

Cell Toxicity Study. The cytotoxicity of HA/CS-CrmA nanoparticles was measured using the 3-(4, 5-dimethylthiazol-2-yl)-2, 5-diphenyltetrazolium bromide (MTT; Sigma-Aldrich) colorimetric assay. Synoviocytes were seeded at a density of $5 \times 10^{4}$ cells per well into 96-well plates $24 \mathrm{~h}$ before the experiment. Increasing doses of HA/CS-CrmA nanoparticles (from $0 \mu \mathrm{g} / \mathrm{mL}$ to $60 \mu \mathrm{g} / \mathrm{mL}$ ) were incubated with the synoviocytes for $5 \mathrm{~h}$, respectively. The HA/CS-CrmA nanoparticles were then discarded and the cells were removed and placed in fresh media. MTT solution $(0.5 \mathrm{mg} / \mathrm{mL})$ was added to the synoviocytes before incubation at $37^{\circ} \mathrm{C}$ with $5 \% \mathrm{CO}_{2}$ for $4 \mathrm{~h}$. The medium was removed, and the formazan crystals formed in each well were dissolved in $100 \mu \mathrm{L}$ dimethyl sulfoxide. Absorbance values were measured at $570 \mathrm{~nm}$ using a microplate reader (model 680; Bio-Rad, Hercules, CA, USA).

\section{KARGER}


In vitro Transfection Experiment. Synoviocytes were seeded at a density of $1 \times 10^{4}$ cells per well into 24-well plates and grown for $24 \mathrm{~h}$ prior to transfection. Cells were washed, and the culture medium was replaced by Hanks' balanced salt solution ( $\mathrm{pH}$ 6.4). HA/CS-CrmA nanoparticles ( $40 \mu \mathrm{g} / \mathrm{mL}$ ) were added to the cells. After $4 \mathrm{~h}$, the medium was replaced with fresh culture medium. Five days post-transfection, the cells were harvested and the expression of CrmA was measured by western blot to confirm the validity of the transfection, as described previously [28].

\section{Animal Studies}

ACLT Model of OA. Male Sprague-Dawley rats ( $\mathrm{n}=48,6-8$ weeks old) were supplied by the animal experiment center of Wuhan University, China, and randomly divided into the following four groups (n = 12/group): (1) sham operated control + saline (Control); (2) ACLT + saline (ACLT); (3) ACLT + HA/CS nanoparticles (ACLT-HA/CS); (4) ACLT + HA/CS-CrmA nanoparticles (ACLT-HA/CS-CrmA). Rats were anesthetized via intraperitoneal injection of sodium pentobarbital ( $45 \mathrm{mg} / \mathrm{kg})$, and their skin was prepped with a topical antiseptic. The skin laterally to the right knee joint was incised to expose the joint capsules. The joint capsules were opened, and the anterior cruciate ligament was transected using a surgical scalpel. Surgery was performed on the right knee joints. Rats in the Control group were subjected to surgical manipulation without ACLT. Intra-articular injections of HA/CS nanoparticles ( $40 \mu \mathrm{g} / \mathrm{mL}, 100 \mu \mathrm{L})$, HA/CSCrmA nanoparticles $(40 \mu \mathrm{g} / \mathrm{mL}, 100 \mu \mathrm{L})$ or equal amounts of saline were performed 4 weeks after ACLT, with each animal receiving one injection every 4 weeks. All animals were euthanized at 12 weeks after ACLT surgery.

Histological Analysis. Samples were cut from the femur $5 \mathrm{~mm}$ from the weight-bearing area of the articular cartilage of ACLT joints and embedded in paraffin. Six sections were stained with Safranin 0/Fast Green to assess cartilage GAG content. Another six sections were stained with hematoxylin and eosin to observe inflammation in the synovial tissues. The Osteoarthritis Research Society International (OARSI) cartilage grading system was used to quantify cartilage degradation and synovial membrane inflammation [29].

Immunohistochemistry. The expression of type II collagen in cartilage was measured by immunohistochemistry. Briefly, paraffin sections were incubated with the anti-type II collagen (Abcam, Cambridge, MA, USA) at $4{ }^{\circ} \mathrm{C}$ overnight. Sections were incubated with a biotinylated secondary antibody and streptavidin-peroxidase conjugate (Invitrogen, Carlsbad, CA, USA), followed by standardized development in 3'3-diaminobenzidine. Sections were visualized using a Nikon E800 microscope (Nikon, Melville, NY, USA). To quantify the optical density, 6 fields at $\times 200$ magnifications were randomly selected from six sections in each group and analyzed using Image-Pro Plus 6.0 (Media Cybernetics, Silver Spring, MD, USA).

Western Blotting. Proteins were extracted from the synovial tissues collected from the ACLT joints of the animals. After the concentrations of the proteins were determined, equal amounts of protein were electrophoresed on sodium dodecyl sulfate-polyacrylamide gel and transferred to polyvinylidene difluoride membranes. The membranes were blocked with 5\% non-fat dry milk in Tis-buffer saline- $0.05 \%$ Tween (pH 7.4) and incubated with anti-IL-1 $\beta,-M M P-3$, and -MMP-13 antibodies (Abcam) at $4{ }^{\circ} \mathrm{C}$ overnight. The membrane was washed and incubated with horseradish peroxidase-conjugated secondary antibody (Boster Biological Technology, Wuhan, China) at $37^{\circ} \mathrm{C}$ for $2 \mathrm{~h}$. The protein bands were visualized by an enhanced chemiluminescence system, and glyceraldehydes-3-phosphate dehydrogenase (GAPDH, Boster) was used as an internal control to correct variation among the different samples.

\section{Statistical Analysis}

All continuous data were expressed as the mean \pm SD and analyzed using SPSS 19.0 (IBM Corp, Chicago, IL, USA). The OARSI scores of cartilage degradation and synovial membrane inflammation were analyzed by Kruskal-Wallis one-way analysis of variance (ANOVA). Other data were analyzed by one-way ANOVA followed by the Bonferroni's correction for post-hoc multiple comparisons. All graphics were created using the GraphPad Prism 6.0 software (GraphPad Software Inc., San Diego, CA, USA). A $P$ value $<0.05$ was considered statistically significant. 


\section{Results}

\section{Characterization of HA/CS-CrmA Nanoparticles}

As shown in Fig. 1A, images of HA/CS-CrmA nanoparticles photographed by SEM displayed an even distribution of particles, most of which had regular spherical shapes and diameters of 100-300 $\mathrm{nm}$.

The degree of protection afforded by HA/CS on pDNA-CrmA was evaluated by agarose gel electrophoresis assays (Fig. 1B). Lane 2 showed that pDNA-CrmA was completely retained within the gel-loading well, which demonstrated the whole combination of pDNA-CrmA with HA/CS nanoparticles. After adding DNase I, the fluorescence signal from lane 5 containing naked pDNA-CrmA disappeared completely, whereas that of lane 4 containing HA/CS-CrmA nanoparticles remained. These results indicated that HA/CS-CrmA entrapped pDNA-CrmA. However, after treatment by chitosanase, the degradation of CS contributed to release of entrapped pDNA-CrmA from the nanoparticles and migrated into the gel (lane 6).

The release profile of pDNA-CrmA from the HA/CS-CrmA nanoparticles is shown in Fig. 1C. On the first day, the release of pDNA-CrmA occurred as a small burst of about 10-20\%. On the remaining days, the release of pDNA-CrmA occurred at a constant slow rate and reached more than $70 \%(71.7 \pm 3.1 \%)$ on day 22 .

\section{Cytotoxicity and Transfection of HA/CS-CrmA Nanoparticles}

The cytotoxicity of the HA/CS-CrmA nanoparticles was measured by MTT assay (Fig. 2A). As the nanoparticles concentration increased to $60 \mu \mathrm{g} / \mathrm{mL}$, the HA/CS-CrmA nanoparticles exhibited a significant increase in cellular toxicity $(P<0.05)$, indicating that HA/CS-CrmA nanoparticles are safe carriers to joint tissue/synoviocytes with a safety range of 0-40 $\mu \mathrm{g} /$ $\mathrm{mL}$.

HA/CS-CrmA nanoparticles were co-cultured with synoviocytes to evaluate the cellular uptake of the HA/CS-CrmA nanoparticles. As shown in Fig. 2B, effective gene transfection was validated by western blot, which showed the expression of CrmA.

\section{Effect of HA/CS-CrmA Nanoparticles on Cartilage Damage Induced by OA}

To confirm the therapeutic effects of HA/CS-CrmA nanoparticles on OA, a rat ACLT modal of OA was established. Cartilage damage was assessed by Safranin O/Fast Green staining, and the expression of type II collagen in cartilage was determined by immunohistochemistry. As shown in Fig. 3A, losses of the superficial layer, cellularity and fibrosis were observed in the ALCT group but not in the Control group at 12 weeks after ACLT. Intra-articular injection of both HA/CS-CrmA nanoparticles and HA/CS nanoparticles attenuated cartilage lesion formation retained more of the superficial layer and led to less fibrosis and more cellularity. However, the cartilage in the ACLT-HA/CS-CrmA group had strong GAG staining and a more intact surface than that of the ACLT-HA/CS group. This result was further confirmed by the OARSI cartilage degradation scores (Fig. 3C). Compared with the Control group, the OARSI score of the ACLT group dramatically increased $(P<0.05)$. Intra-articular injection of either

Fig. 1. Characterization of HA/ CS-CrmA nanoparticles. (A) Representative image of HA/CS-CrmA nanoparticles obtained by SEM (10000× magnification). (B) Gel electrophoresis of HA/CS-CrmA nanoparticles, digests, and control samples. Lane 1, DNA marker;

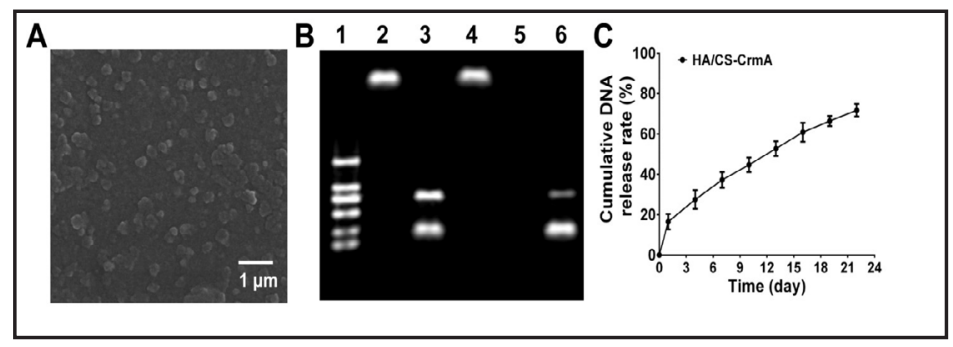
Lane 2, HA/CS-CrmA nanoparticles; Lane 3, normal naked pDNA-CrmA; Lane 4, HA/CS-CrmA nanoparticles digested by DNase I; Lane 5, naked pDNA-CrmA digested by DNase I; Lane 6, HA/CS-CrmA nanoparticles digested by chitosanase. (C) Cumulative pDNA release profile of HA/CS-CrmA nanoparticles as a function of time up to 22 days. 
Zhou et al.: HA/CS-CrmA Nanoparticles Inhibit Cartilage Destruction and Synovial Inflammation Induced by ACLT

Fig. 2. Cytotoxicity and transfection of HA/CS-CrmA nanoparticles. (A) Cell viability of HA/CS- CrmA nanoparticles at different doses. (B) The expression of CrmA detected by western blot demonstrated that HA/CS-CrmA nanoparticles effectively transfected synoviocytes. ${ }^{*} \mathrm{P}<0.05$ vs. $0 \mu \mathrm{g} / \mathrm{mL}$.

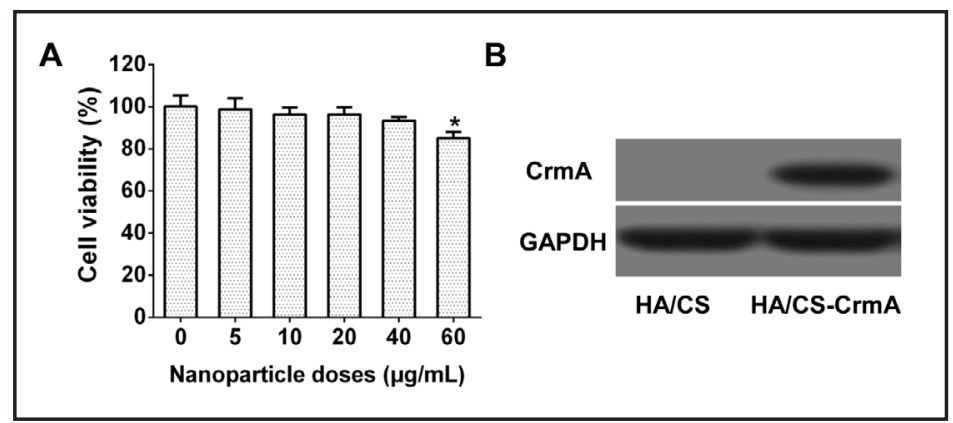

Fig. 3. Effect of HA/CS-CrmA nanoparticles on cartilage damage induced by OA. (A) Representative images of the articular surfaces stained with safranin $0 /$ fast green among groups (200× magnification). (B) Representative images of immunohistochemical staining displaying the expression level of type II collagen in cartilage $(200 \times$ magnification). (C) OARSI scores of cartilage degradation $(n=6)$. (D) The mean optical density of type II collagen among the groups $(\mathrm{n}=$ 6). ${ }^{*} \mathrm{P}<0.05$ vs. the Control group;

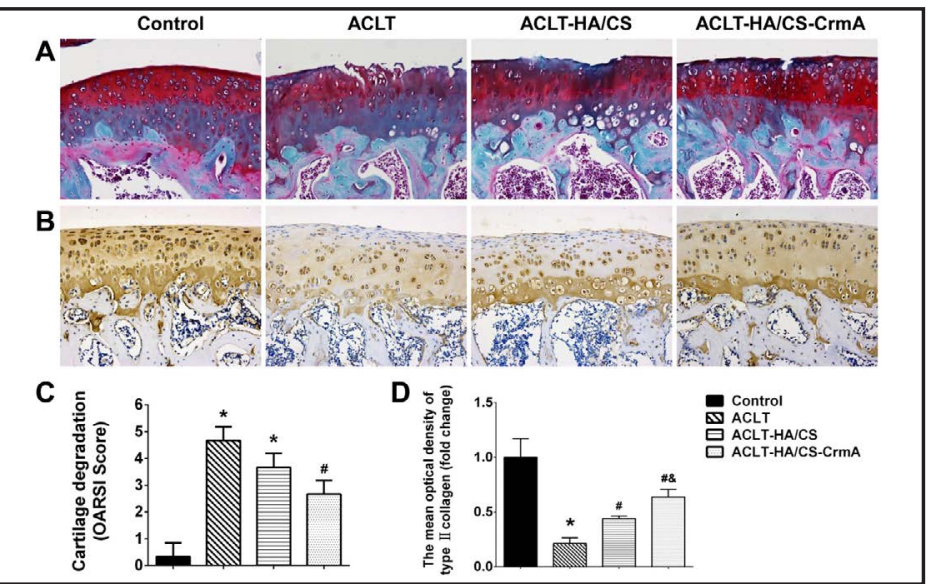
${ }^{\#} \mathrm{P}<0.05$ vs. the ACLT group; ${ }^{\circledR} \mathrm{P}<0.05$ vs. the ACLT-HA/CS group.

HA/CS nanoparticles or HA/CS-CrmA nanoparticles decreased the OARSI score. However, a statistically significant difference was found in only the ACLT-HA/CS-CrmA group.

Immunohistochemistry revealed that the mean optical density of type II collagen in cartilage was significantly decreased in the ALCT group when compared with the Control group $(P<0.05)$. Intra-articular injection of HA/CS nanoparticles or HA/CS-CrmA nanoparticles both significantly suppressed the decrease of the mean optical density of type II collagen in cartilage induced by ACLT. However, the effect of the HA/CS-CrmA nanoparticles was stronger than that of the HA/CS nanoparticles $(P<0.05$, Fig. 3D).

\section{Effect of HA/CS-CrmA Nanoparticles on Synovial Inflammation Induced by OA}

At 12 weeks after ACLT, synovial inflammation was measured by hematoxylin and eosin staining and the expression levels of IL-1 $\beta$, MMP-3, and MMP-13 in synovial tissue were detected by western blot. As shown in Fig. 4A, the Control group showed normal synovial tissue, whereas infiltration of inflammatory cells and increased numbers of synovial lining cell layers were observed in the ACLT group. Treatment with either HA/CS-CrmA or HA/ CS nanoparticles displayed less inflammatory cell infiltration and decreased numbers of synovial lining cell layers, but the effect of HA/CS-CrmA nanoparticles seemed stronger than that of the HA/CS nanoparticles. Similar results were confirmed by the OARSI scores of synovial membrane inflammation (Fig. 4B). The OARSI score of the ACLT group dramatically increased compared with the Control group $(P<0.05)$. Intra-articular injection of either HA/CS nanoparticles or HA/CS-CrmA nanoparticles decreased the OARSI scores. However, a significant difference was found only in the ACLT-HA/CS-CrmA group.

The results of western blot showed that the expression levels of IL-1 $\beta$, MMP-3 and MMP13 in synovial tissue were significantly increased in the ACLT group when compared with the Control group $(P<0.05)$. Intra-articular injection of HA/CS nanoparticles or HA/CS-CrmA nanoparticles dramatically inhibited the increased expression of IL-1 $\beta$, MMP-3, and MMP-13 
Fig. 4. Effect of HA/CS-CrmA nanoparticles on synovial inflammation induced by OA. (A) Representative images of the synovial tissue stained with hematoxylin and eosin among groups (200x magnification). (B) OARSI scores of synovial membrane inflammation $(n=6) .(C)$ The relative expression levels of IL-1 $\beta$, MMP-3, and MMP13 in synovial tissue measured by western blot $(\mathrm{n}=6)$. ${ }^{*} \mathrm{P}<0.05$ vs.

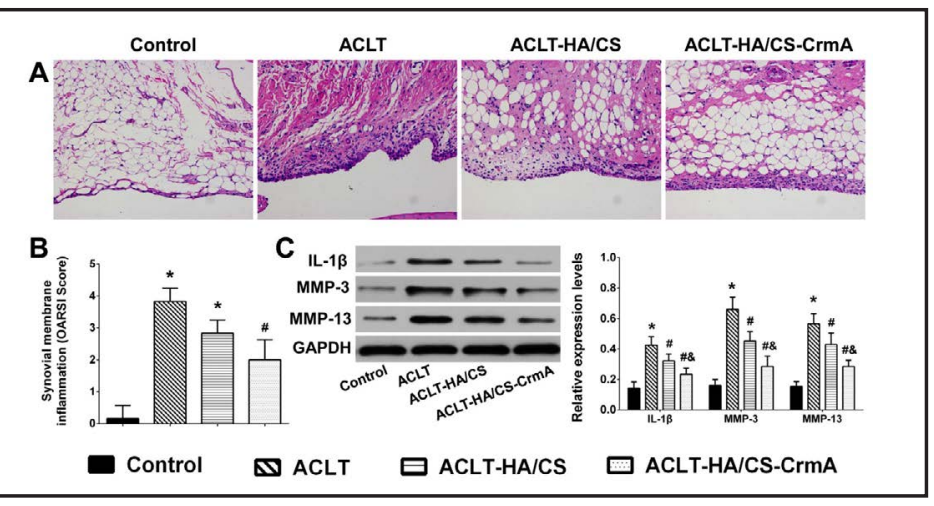
the Control group; ${ }^{\#} \mathrm{P}<0.05$ vs. the ACLT group; ${ }^{\mathrm{P}}<0.05$ vs. the ACLT-HA/CS group.

induced by ACLT $(P<0.05)$. However, the inhibitory effect of HA/CS-CrmA nanoparticles was much stronger than that of HA/CS nanoparticles $(P<0.05$, Fig. $4 \mathrm{C})$.

\section{Discussion}

We constructed a non-viral gene delivery system composed of HA-CS with plasmid DNA encoding CrmA and intended to evaluate its potential effects on OA. The results showed that delivery of pDNA from the HA/CS-CrmA nanoparticles was steady and maintained for more than 22 days in vitro. HA/CS-CrmA nanoparticles were also proved to be safe gene carriers for synoviocyte transfection. Moreover, using a rat ACLT model of OA, we found that HA/CSCrmA nanoparticles significantly attenuated cartilage damage and the loss of type II collagen in cartilage. Meanwhile, intra-articular injection of HA/CS-CrmA nanoparticles markedly inhibited synovial inflammation and the expression levels of IL-1 $\beta$, MMP-3, and MMP-13 in the synovium.

In the treatment of $\mathrm{OA}$, the target protein can be delivered via the following techniques: (1) directly inject the target protein into joint, (2) delivery of protein of interest by a separate device, such as microspheres or microcapsules, (3) entrap the protein or load transfected cells on porous scaffolds for sustained release of the target protein, or (4) deliver plasmid DNA encoding the protein of interest to the joint by viral or non-viral vectors or gene-activated matrices [30-32]. Among these options, a non-viral gene delivery system with encapsulated pDNA encoding the target protein seems to have more advantages over the others. In this system, pDNA has a more stable, flexible chemical properties than protein in vivo and can be used for sustained release [33]. Moreover, it is easy to control the local expression and reduces the need for repeated intra-articular injections [33]. Additionally, non-viral vectors possess superior properties such as high level of safety, low immunogenicity and convenient application [34]. In the present study, we used non-viral vectors-CS-HA-as biomaterials to fabricate nanoparticles to entrap the therapeutic pDNA-CrmA. The gel electrophoresis results of our study indicated that pDNA-CrmA was successfully entrapped in HA/CS-CrmA nanoparticles. In addition, the pDNA-CrmA release profile of nanoparticles showed that HA/ CS-CrmA nanoparticles were well-controlled systems capable of releasing pDNA-CrmA for a lengthy period of more than 22 days, which was much longer than CS/HA microspheres containing protein CrmA that had a release time of over 10 days [25].

$\mathrm{CS}$, a natural copolymer of D-glucosamine and $\mathrm{N}$-acetylglucosamine, has been widely used in various applications, such as drugs [35], DNA delivery [36], and tissue engineering [37]. Zhang et al. showed that nanoparticles prepared with CS and p-DNA could transfect chondrocytes both in vitro and in vivo [14]. However, the relatively low transfection levels have limited their application as ideal gene transfection vectors. It has been reported that 
the transfection efficiency of CS could be improved by combining CS with cationic or anionic biopolymers, such as polyethylenimine [38] or arginine [15]. Lu et al. reported that HA, a biocompatible anionic biopolymer, also improved the transfection efficiency of CS with pDNA in chondrocytes [24]. HA binds to CD 44, which is highly expressed on the cell surfaces of inflamed chondrocytes and synoviocytes and may increase nanoparticle uptake in the osteoarthritic disease state $[20,24]$. In the present study, our data demonstrated that CS/ HA-CrmA nanoparticles could safely transfect synoviocytes. Similar results were shown in the previous study, which found that HA/CS/pDNA nanoparticles encoding transforming growth factor- $\beta 1$ effectively transfected chondrocytes [24].

IL-1 $\beta$ plays an important role in inducing synovial inflammation, which is involved in the early stages of OA pathogenesis $[4,39]$. Elevated levels of IL-1 $\beta$ are observed in the serum and synovial fluids of patients with OA [40]. IL-1 $\beta$ in synovial fluid is associated with an increased risk of chondral damage in knee OA induced by chronic anterior cruciate ligament deficiency [41]. Moreover, IL-1 $\beta$ can activate the synthesis and release of MMPs, such as MMP-3 and MMP-13, which lead to matrix breakdown [42]. It has been reported that the inhibition of IL-1 $\beta$ by an IL-1 receptor antagonist could protect cartilage degeneration as shown in a mouse model of OA [43]. These studies indicate that IL-1 $\beta$ contributes to the pathogenesis and progression of $\mathrm{OA}$, and thus the inhibition of IL-1 $\beta$ in synovial tissue is a promising way to prevent and treat OA. IL- $1 \beta$ converting enzyme (caspase- 1 ) is responsible for cleaving and thereby activating IL-1 $\beta[11,44]$. CrmA, a natural inhibitor of caspase- 1 , can rapidly combine with caspase- 1 and form a tight complex, thus preventing the generation of active IL-1 $\beta[11,12]$. Our previous study showed that the controlled release of CrmA from HA/CS microspheres markedly suppressed the expression level of IL-1 $\beta$ and maintained the phenotype of chondrocytes in vitro [25]. Consistent with the previous study, our data revealed that intra-articular injection of HA/CS-CrmA nanoparticles reduced cartilage damage and the loss of type II collagen in cartilage. Meanwhile, synovial inflammation and the expression of IL-1 $\beta$, MMP-3, and MMP-13 in synovial tissue were dramatically suppressed by HA/CS-CrmA nanoparticles. These findings suggested that HA/CS-CrmA nanoparticles might attenuate cartilage destruction and protect against early $\mathrm{OA}$ by inhibiting synovial inflammation via the inhibition of IL-1 $\beta$ generation.

\section{Conclusion}

The present study demonstrated the feasibility of using a non-viral gene delivery system composed of HA-CS for the expression of CrmA gene. This system provided pDNA release over 3 weeks and helped to raise the amount of CrmA to a high level in a relatively short time. Moreover, the inhibition of IL-1 $\beta$ in synovial tissue by HA/CS-CrmA nanoparticles attenuated cartilage damage and synovial inflammation in a rat model of OA. These results indicated that HA/CS-CrmA nanoparticles could be manipulated to provide localized delivery in the joint space to prevent the progression of OA by inhibiting IL-1 $\beta$ generation in the synovium.

\section{Acknowledgements}

This research was supported by National Natural Science Foundation of China (No. 81501921), Natural Science Foundation of Hubei Province of China (No. 2016CFB500), Wuhan Science and Technology Project of China (No. 2016060101010045), Hubei Provincial Science and Technology Support Program of China (No.2015BCA316).

\section{Disclosure Statement}

The authors declare to have no competing interests. 


\section{Cellular Physiology Cell Physiol Biochem 2018;47:1207-1216 \begin{tabular}{l|l} 
and Biochemistry & $\begin{array}{l}\text { DOI: 10.1159/000490217 } 2018 \text { The Author(s). Published by S. Karger AG, Basel } \\
\text { www.karger.com/cpb }\end{array}$
\end{tabular}

Zhou et al.: HA/CS-CrmA Nanoparticles Inhibit Cartilage Destruction and Synovial Inflammation Induced by ACLT

\section{References}

- Emery CA, Roos EM, Verhagen E, Finch CF, Bennell KL, Story B, Spindler K, Kemp J, Lohmander LS: OARSI Clinical Trials Recommendations: Design and conduct of clinical trials for primary prevention of osteoarthritis by joint injury prevention in sport and recreation. Osteoarthritis Cartilage 2015;23:815-825.

-2 Pelletier JP, Martel-Pelletier J, Abramson SB: Osteoarthritis, an inflammatory disease: potential implication for the selection of new therapeutic targets. Arthritis Rheum 2001;44:1237-1247.

3 Chen S, Zhang L, Xu R, Ti Y, Zhao Y, Zhou L, Zhao J: TheBDKRB2 +9/-9 Polymorphisms Influence ProInflammatory Cytokine Levels in Knee Osteoarthritis by Altering TLR-2 Expression: Clinical and in vitro Studies. Cell Physiol Biochem 2016;38:1245-1256.

4 Smith MD, Triantafillou S, Parker A, Youssef PP, Coleman M: Synovial membrane inflammation and cytokine production in patients with early osteoarthritis. J Rheumatol 1997;24:365-371.

-5 Attur MG, Patel IR, Patel RN, Abramson SB, Amin AR: Autocrine production of IL-1 beta by human osteoarthritis-affected cartilage and differential regulation of endogenous nitric oxide, IL-6, prostaglandin E2, and IL-8 Proc Assoc Am Physicians 1998;110:65-72.

6 Mao G, Wu P, Zhang Z, Zhang Z, Liao W, Li Y, Kang Y: MicroRNA-92a-3p Regulates Aggrecanase-1 and Aggrecanase-2 Expression in Chondrogenesis and IL-1beta-Induced Catabolism in Human Articular Chondrocytes. Cell Physiol Biochem 2017;44:38-52.

7 Daheshia M, Yao JQ: The interleukin 1beta pathway in the pathogenesis of osteoarthritis. J Rheumatol 2008;35:2306-2312.

-8 Santangelo KS, Nuovo GJ, Bertone AL: In vivo reduction or blockade of interleukin-1beta in primary osteoarthritis influences expression of mediators implicated in pathogenesis. Osteoarthritis Cartilage 2012;20:1610-1618.

-9 Chevalier X, Conrozier T, Richette P: Desperately looking for the right target in osteoarthritis: the anti-IL-1 strategy. Arthritis Res Ther 2011;13:124.

10 Sachs D, Cunha FQ, Poole S, Ferreira SH: Tumour necrosis factor-alpha, interleukin-1beta and interleukin-8 induce persistent mechanical nociceptor hypersensitivity. Pain 2002;96:89-97.

-11 Ray CA, Black RA, Kronheim SR, Greenstreet TA, Sleath PR, Salvesen GS, Pickup DJ: Viral inhibition of inflammation: cowpox virus encodes an inhibitor of the interleukin-1 beta converting enzyme. Cell 1992;69:597-604.

12 Komiyama T, Ray CA, Pickup DJ, Howard AD, Thornberry NA, Peterson EP, Salvesen G: Inhibition of interleukin-1 beta converting enzyme by the cowpox virus serpin CrmA. An example of cross-class inhibition. J Biol Chem 1994;269:19331-19337.

13 Zhou PH, Shi L, Qiu B: Protective effect of controlled release of cytokine response modifier A from chitosan microspheres on rat chondrocytes from interleukin-1beta induced inflammation and apoptosis. Exp Ther Med 2017;14:3170-3178.

14 Zhang X, Yu C, Xushi, Zhang C, Tang T, Dai K: Direct chitosan-mediated gene delivery to the rabbit knee joints in vitro and in vivo. Biochem Biophys Res Commun 2006;341:202-208.

15 Gao Y, Xu Z, Chen S, Gu W, Chen L, Li Y: Arginine-chitosan/DNA self-assemble nanoparticles for gene delivery: In vitro characteristics and transfection efficiency. Int J Pharm 2008;359:241-246.

$>16$ Hejazi R, Amiji M: Chitosan-based gastrointestinal delivery systems. J Control Release 2003;89:151-165.

17 Li Z, Zhang M: Chitosan-alginate as scaffolding material for cartilage tissue engineering. J Biomed Mater Res A 2005;75:485-493.

-18 Menzel EJ, Farr C: Hyaluronidase and its substrate hyaluronan: biochemistry, biological activities and therapeutic uses. Cancer Lett 1998;131:3-11.

19 Almond A: Hyaluronan. Cell Mol Life Sci 2007;64:1591-1596.

20 Campo GM, Avenoso A, Campo S, D’Ascola A, Nastasi G, Calatroni A: Small hyaluronan oligosaccharides induce inflammation by engaging both toll-like- 4 and CD44 receptors in human chondrocytes. Biochem Pharmacol 2010;80:480-490.

-21 Salter DM, Godolphin JL, Gourlay MS, Lawson MF, Hughes DE, Dunne E: Analysis of human articular chondrocyte CD44 isoform expression and function in health and disease. J Pathol 1996;179:396-402.

22 Zhang FJ, Luo W, Gao SG, Su DZ, Li YS, Zeng C, Lei GH: Expression of CD44 in articular cartilage is associated with disease severity in knee osteoarthritis. Mod Rheumatol 2013;23:1186-1191.

23 Zhang FJ, Gao SG, Cheng L, Tian J, Xu WS, Luo W, Song Y, Yang Y, Lei GH: The effect of hyaluronic acid on 


\section{Cellular Physiology Cell Physiol Biochem 2018;47:1207-1216 \begin{tabular}{l|l|l} 
DOI: 10.1159/000490217 & $\begin{array}{l}\text { (c) } 2018 \text { The Author(s). Published by S. Karger AG, Basel } \\
\text { www.karger.com/cpb }\end{array}$
\end{tabular}

Zhou et al.: HA/CS-CrmA Nanoparticles Inhibit Cartilage Destruction and Synovial Inflammation Induced by ACLT

osteopontin and CD44 mRNA of fibroblast-like synoviocytes in patients with osteoarthritis of the knee. Rheumatol Int 2013;33:79-83.

24 Lu HD, Zhao HQ, Wang K, Lv LL: Novel hyaluronic acid-chitosan nanoparticles as non-viral gene delivery vectors targeting osteoarthritis. Int J Pharm 2011;420:358-365.

-25 Ma BL, Zhou PH, Xie T, Shi L, Qiu B, Wang Q: Inhibition of interleukin-1beta-stimulated dedifferentiation of chondrocytes via controlled release of CrmA from hyaluronic acid-chitosan microspheres. BMC Musculoskelet Disord 2015;16:61.

-26 Ravina M, Cubillo E, Olmeda D, Novoa-Carballal R, Fernandez-Megia E, Riguera R, Sanchez A, Cano A, Alonso MJ: Hyaluronic acid/chitosan-g-poly(ethylene glycol) nanoparticles for gene therapy: an application for pDNA and siRNA delivery. Pharm Res 2010;27:2544-2555.

27 Close B, Banister K, Baumans V, Bernoth EM, Bromage N, Bunyan J, Erhardt W, Flecknell P, Gregory N, Hackbarth H, Morton D, Warwick C: Recommendations for euthanasia of experimental animals: Part 2 DGXT of the European Commission. Lab Anim 1997;31:1-32.

28 Fujino M, Li XK, Suda T, Hashimoto M, Okabe K, Yaginuma H, Mikoshiba K, Guo L, Okuyama T, Enosawa S, Amemiya H, Amano T, Suzuki S: In vitro prevention of cell-mediated xeno-graft rejection via the Fas/FasLpathway in CrmA-transducted porcine kidney cells. Xenotransplantation 2001;8:115-124.

29 Gerwin N, Bendele AM, Glasson S, Carlson CS: The OARSI histopathology initiative - recommendations for histological assessments of osteoarthritis in the rat. Osteoarthritis Cartilage 2010;18 Suppl 3:S24-34.

-30 Edelman ER, Mathiowitz E, Langer R, Klagsbrun M: Controlled and modulated release of basic fibroblast growth factor. Biomaterials 1991;12:619-626.

31 Camarata PJ, Suryanarayanan R, Turner DA, Parker RG, Ebner TJ: Sustained release of nerve growth factor from biodegradable polymer microspheres. Neurosurgery 1992;30:313-319.

-32 Tabata Y, Hijikata S, Muniruzzaman M, Ikada Y: Neovascularization effect of biodegradable gelatin microspheres incorporating basic fibroblast growth factor. J Biomater Sci Polym Ed 1999;10:79-94.

-33 Guo T, Zhao J, Chang J, Ding Z, Hong H, Chen J, Zhang J: Porous chitosan-gelatin scaffold containing plasmid DNA encoding transforming growth factor-beta1 for chondrocytes proliferation. Biomaterials 2006;27:1095-1103.

-34 Oligino TJ, Yao Q, Ghivizzani SC, Robbins P: Vector systems for gene transfer to joints. Clin Orthop Relat Res 2000;S17-30.

-35 Lee JE, Kim KE, Kwon IC, Ahn HJ, Lee SH, Cho H, Kim HJ, Seong SC, Lee MC: Effects of the controlledreleased TGF-beta 1 from chitosan microspheres on chondrocytes cultured in a collagen/chitosan/ glycosaminoglycan scaffold. Biomaterials 2004;25:4163-4173.

-36 Wu H, Wang S, Fang H, Zan X, Zhang J, Wan Y: Chitosan-polycaprolactone copolymer microspheres for transforming growth factor-beta1 delivery. Colloids Surf B Biointerfaces 2011;82:602-608.

-37 Suh JK, Matthew HW: Application of chitosan-based polysaccharide biomaterials in cartilage tissue engineering: a review. Biomaterials 2000;21:2589-2598.

-38 Zhao QQ, Chen JL, Lv TF, He CX, Tang GP, Liang WQ, Tabata Y, Gao JQ: N/P ratio significantly influences the transfection efficiency and cytotoxicity of a polyethylenimine/chitosan/DNA complex. Biol Pharm Bull 2009;32:706-710.

-39 Goldring MB, Otero M: Inflammation in osteoarthritis. Curr Opin Rheumatol 2011;23:471-478.

-40 Hussein MR, Fathi NA, El-Din AM, Hassan HI, Abdullah F, Al-Hakeem E, Backer EA: Alterations of the CD4(+), CD8 (+) T cell subsets, interleukins-1beta, IL-10, IL-17, tumor necrosis factor-alpha and soluble intercellular adhesion molecule-1 in rheumatoid arthritis and osteoarthritis: preliminary observations. Pathol Oncol Res 2008;14:321-328.

41 Marks PH, Donaldson ML: Inflammatory cytokine profiles associated with chondral damage in the anterior cruciate ligament-deficient knee. Arthroscopy 2005;21:1342-1347.

42 Kobayashi M, Squires GR, Mousa A, Tanzer M, Zukor DJ, Antoniou J, Feige U, Poole AR: Role of interleukin-1 and tumor necrosis factor alpha in matrix degradation of human osteoarthritic cartilage. Arthritis Rheum 2005;52:128-135.

43 Zhang B, Hu J, Man C, Zhu S: Effect of intra-articular administration of interleukin 1 receptor antagonist on cartilage repair in temporomandibular joint. J Craniofac Surg 2011;22:711-714.

44 Cerretti DP, Kozlosky CJ, Mosley B, Nelson N, Van Ness K, Greenstreet TA, March CJ, Kronheim SR, Druck T, Cannizzaro LA, et al.: Molecular cloning of the interleukin-1 beta converting enzyme. Science 1992;256:97100. 